\title{
A Discrete-Time Markov Chain Based Comparison of the MAC Layer Performance of C-V2X Mode 4 and IEEE 802.11p
}

\author{
Geeth P. Wijesiri N.B.A, Member, IEEE, Jussi Haapola, Member, IEEE, and Tharaka Samarasinghe, Senior \\ Member, IEEE
}

\begin{abstract}
Vehicle-to-vehicle ( V2V) communication plays a pivotal role in intelligent transport systems (ITS) with cellularvehicle to everything (C-V2X) and IEEE 802.11p being the two competing enabling technologies. This paper presents multidimensional discrete-time Markov chain (DTMC) based models to study the medium access control (MAC) layer performance of the IEEE 802.11p standard and C-V2X Mode 4, considering periodic cooperative awareness messages (CAMs) and event-driven decentralized environmental notification messages (DENMs). Closed-form solutions for the models' steady-state probabilities are obtained, which are then utilized to derive expressions for several key performance metrics. Numerical results are provided to draw insights on the performance. In particular, a performance comparison between IEEE 802.11p and C-V2X Mode 4 in terms of the average delay, the collision probability, and the channel utilization is presented. The results show that IEEE 802.11p is superior in terms of average delay, whereas C-V2X Mode 4 excels in collision resolution. The paper also includes design insights on possible future MAC layer performance enhancements of both standards.
\end{abstract}

Index Terms-C-V2X Mode 4, discrete-time Markov chain, ETSI ITS-G5, IEEE 802.11p, medium access control, vehicle-tovehicle communication.

\section{INTRODUCTION}

\section{A. Background and Motivation}

$\mathbf{V}$ EHICLE to everything (V2X) communication is envisioned to be a major technological advancement that shapes our future mobility and quality of life. Vehicular networks primarily depend on V2X communications in enabling an active safety environment. To this end, IEEE $802.11 \mathrm{p}$ / dedicated short-range communication (DSRC) is known to be the first commercial V2X communication technology.

G. P. Wijesiri N.B.A is with the Department of Electronic and Telecommunication Engineering, University of Moratuwa, Sri Lanka, the Center for Wireless Communication, University of Oulu, Finland, and the Department of Electrical and Information Engineering, University of Ruhuna, Sri Lanka (e-mail: geeth@eie.ruh.ac.lk).

J. Haapola is with the Center for Wireless Communication, University of Oulu, Finland (e-mail: jussi.haapola@oulu.fi).

T. Samarasinghe is with the Department of Electronic and Telecommunication Engineering, University of Moratuwa, Sri Lanka, and the Department of Electronic and Electrical Engineering, University of Melbourne, Australia (e-mail: tharakas@uom.lk).

This research has been partially financially supported by the ITEA 3 project APPSTACLE (15017), the Academy of Finland 6Genesis Flagship (grant 318927) and the AHEAD/RA3/RIC/MRT/ITS-Multidisciplinary Transport Development Project of the University of Moratuwa under World Bank grant 6026-LK/8743-LK.

The material in this paper was presented in part at the IEEE 90th Vehicular Technology Conference, Hawaii, USA, September 2019.
The first WiFi-based standard specifically designed for vehicular communications was approved under IEEE 802.11p in 2010 [1], later included in IEEE 802.11-2012 [2], and now superseded by IEEE 802.11-2016 [3]. The European Telecommunications Standards Institute's intelligent transport systems operating in the $5 \mathrm{GHz}$ frequency band (ETSI ITSG5) [4]-[7] subsequently has been approved as the European version of the IEEE $802.11 \mathrm{p}$ standard.

As an alternative to IEEE $802.11 \mathrm{p}$, the third generation partnership project (3GPP) included support for V2X communications using long-term evolution (LTE) sidelink communications, a.k.a., LTE-V, LTE-V2X, LTE-V2V, or Cellular-V2X (C-V2X). The LTE sidelink was introduced for public safety device-to-device (D2D) communications in Release 12 as Mode 1 and Mode 2. Release 14 introduced Mode 3 and Mode 4, specifically designed for V2X communications [8], [9]. Mode 3 enables direct communication between two vehicles, but the selection and management of the radio resources are taken care by the cellular infrastructure. C-V2X Mode 4 on the other hand has many operational similarities with $802.11 \mathrm{p}$. Both technologies facilitate the vehicles to autonomously select and manage their resources and communicate without any infrastructure support. In this paper, we focus on ETSI ITS-G5 802.11p and C-V2X Mode 4.

Vehicular communication mainly consists of an exchange of small broadcast packets with critical latency and reliability constraints. To this end, cooperative awareness messages (CAM) and decentralized environmental notification messages (DENM) are two types of broadcast packets used by both IEEE $802.11 \mathrm{p}$ and C-V2X Mode 4 in enabling effective communication and ensuring safety. Information related to cooperative awareness, such as position, dynamics, and attributes, is packed in the periodically transmitted CAM packets [4]. On the other hand, DENM are event-driven messages, triggered by random events such as sudden human-initiated disturbances to the vehicle's pattern of motion (e.g., lane changing, signal violation, emergency braking, road-works), and events caused by weather or nature [5]. The broadcast nature, and the strict latency and reliability constraints of the packets make the medium access control (MAC) layer performance of these technologies crucial, which triggered our motivation for this study.

The MAC layer operations of the two competing technologies are significantly different from one another. The multiple access technique in IEEE $802.11 \mathrm{p}$ is a version of the well- 
known carrier sense multiple access with collision avoidance (CSMA/CA). The contention-based protocol requires a vehicle to sense the medium and check if it is idle before transmitting. A mechanism based on random backoff is executed to reduce the probability of collisions. On the other hand, C-V2X Mode 4 utilizes a distributed sensing-based scheduling protocol called semi-persistent scheduling (SPS) [10]. Vehicles sense and keep track of the previous transmissions of all neighboring vehicles to estimate free resources and pick a free resource for transmission to avoid packet collisions. Our paper primarily focuses on analytically modeling the MAC layer protocols of C-V2X Mode 4 and IEEE 802.11p by utilizing discrete-time Markov chains (DTMCs).

\section{B. Related Works}

Several works have recently discussed the two technologies from various perspectives, mainly focusing on the physical (PHY) layer, with some providing performance comparisons as well [11]-[19]. The MAC layer performance has been studied in [20]-[23]. The first analytical model for the MAC layer performance of C-V2X Mode 4 is proposed in [21]. The paper considers a PHY layer model to capture the effect of the distance between a transmitting node and a receiving node, and the SPS algorithm for resource allocation. The authors then obtain analytical expressions for key MAC layer performance metrics as a function of the distance between the transmitter and the receiver. The first DTMC based analytical model for $\mathrm{C}-\mathrm{V} 2 \mathrm{X}$ Mode 4 is presented by the authors in [22].

In addition to the model for C-V2X Mode 4 presented in [22], this paper also presents a DTMC based analytical model for the MAC layer operations of IEEE 802.11p, which facilitates a comparison of these two technologies. The proposed model in this paper improves the DTMC for IEEE 802.11p presented in [23] along multiple facets. The main novelty is the higher modeling granularity (resolution). The improved modeling granularity allows us to study the whole protocol operation at the aSlotTime level, which is the smallest time unit of $13 \mu$ s defined in the standard. The representation also in turn leads to a fair comparison with our model for $\mathrm{C}-\mathrm{V} 2 \mathrm{X}$ Mode 4, that can be studied at the smallest time unit in its standard, called the subframe (1 ms). Additionally, the model in our paper, captures the effect of the arbitration inter-frame spacing (AIFS) duration, which is an important parameter used in IEEE 802.11p when dealing with multi-priority data streams. Thus, the model in our paper is significantly different from the one in [23].

Modeling the packet generation is another important aspect of a study on MAC layer performance. To this end, [21] utilizes a packet generator with a fixed inter-arrival rate, generating packets with similar characteristics to CAM. The authors of [23] utilize a simple Bernoulli process for the random packet generator to model event-driven packet generation such as DENM. However, such a generator omits periodic traffic such as CAM, and also the periodic re-transmission of DENM packets, which is done for added reliability [24]. Another novel aspect of our work is implementing separate DTMC models for CAM (synchronous) and DENM (asynchronous) packet generation, intending to create a more realistic V2X communication environment. With these novel traffic generators, the system can be modeled for more complex and realistic traffic arrival patterns than the packet generators found in the literature.

\section{Contributions}

The main contributions of our paper can be summarized as follows:

- Analytical modeling: We provide detailed modeling of the MAC layer protocols of C-V2X Mode 4 and ETSI ITS-G5 IEEE 802.11p by utilizing DTMCs. The complete Markov model consists of a DTMC each for the MAC layer operations of the two competing technologies, two DTMCs to model the generation of CAM and DENM packets, and a queue model to represent a device level packet queue.

- Derivation of performance metrics: We obtain closedform expressions for the steady-state probabilities of the DTMCs, which are then used to derive expressions for key performance metrics such as the average delay, the collision probability, and the channel utilization of a vehicular network.

- Numerical comparison of performance: We present an application of the models to provide further insights and comparisons on the derived performance indicators through numerical evaluations. In particular, we show that C-V2X Mode 4 exhibits a lower collision probability compared to IEEE 802.11p, but IEEE 802.11p maintains a lower average delay compared to C-V2X Mode 4.

- Design insights for performance enhancement: Design insights on how the MAC layer performance of both technologies can be improved are presented. These insights can be utilized for future releases and evolution into new radio V2X (NR-V2X) and IEEE 802.11bd [25], [26].

The remainder of the paper is organized as follows. The analytical models and the steady-state solutions are presented in Sections II and III, respectively. Section IV consists of the performance analysis. The numerical results and discussion follow in Section V, and Section VI concludes this paper.

\section{ANALYTICAL MODELS}

This section presents five DTMCs that are dependent on each other. Firstly, we use two DTMCs to model the generation of CAM and DENM packets. We refer to them as packet generators. The third DTMC models the device level packet queue of a vehicle that consists of the generated CAM and DENM packets. The remaining two DTMCs model the MAC layer operations of C-V2X Mode 4 and ETSI ITS-G5 IEEE 802.11 p, respectively. A holistic view of the overall model that consists of these DTMCs is illustrated in Fig. 1, while also showing how they are interrelated. The parameters that lead to the dependence among the DTMCs will be formally introduced later in the section, while presenting the individual DTMCs.

All DTMCs ensure that there is a sequence of transitions of non-zero probability from any state to another (irreducible), 


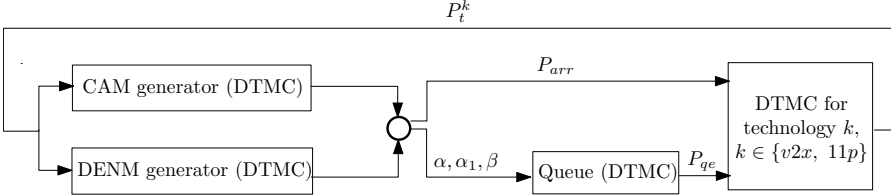

Figure 1: Flowchart illustrating the total model and the dependence among the individual DTMCs.

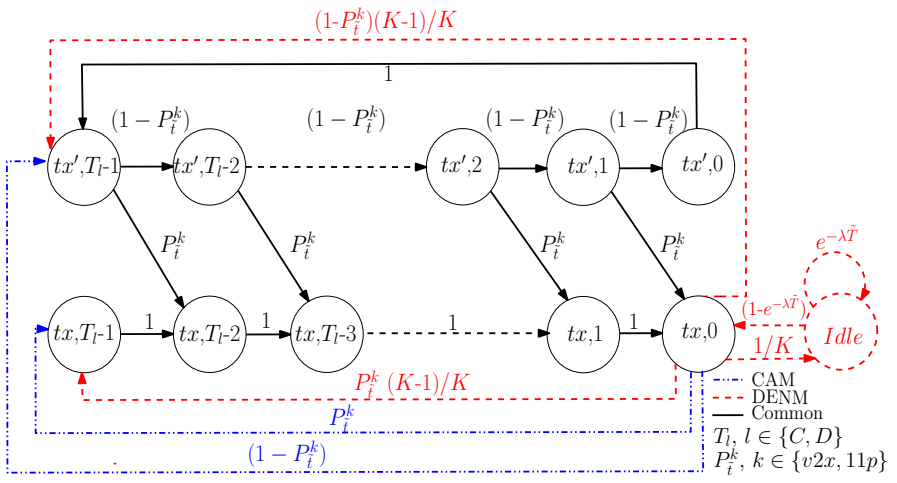

Figure 2: DTMC models for CAM and DENM packet generation, where the line styles are used to differentiate the two models.

and that the states are not partitioned into sets such that all state transitions occur cyclically from one set to another (aperiodic). Thus, the DTMCs are ergodic, and hence, a steady-state distribution exists [27]. The models are based on non-saturation conditions with regards to transmission, i.e., they consider situations when there are no packets to transmit as well, making them more realistic compared to models that assume continuous transmission of packets (saturation conditions). However, the models do not account for a real received power based sensing mechanism. Thus the impact of relative distance, exposed and hidden terminals, are omitted in this study.

\section{A. Packet Generator and Queue Models}

The generator models of CAM and DENM share significant similarities. Therefore, we use a single figure (Fig. 2) to illustrate the DTMCs of the two generator models. The solid lines (black) are used to represent the states and transitions common to the state spaces of both models, and the states and transitions unique to the generation of a particular type of packet are differentiated using line styles and colors. The periodic CAM packet generation is modeled using a fixed inter-arrival time model, where the inter-arrival time $T_{C}$ is set between $100 \mathrm{~ms}$ and $1000 \mathrm{~ms}$ according to the standard [4]. DENM, on the other hand, are random event-driven messages that are not periodic. Thus, an additional idle state (Idle) is included in the DENM generator to capture the periods with no DENM packet generation. Furthermore, DENM are generated on the observation of random events, thus the packet generation is random. Using a Poisson arrival process for such random packet generation is common in the literature on queuing theory (e.g., the arrival of calls at an exchange), and the same has been implemented in [28] for DENM packets. Therefore, we model the triggered arrivals of DENM packets using a Poisson process of intensity $\lambda$ packet/s, by assuming that the triggering events are independent of each other and do not occur simultaneously. Thus, the probability of at least one

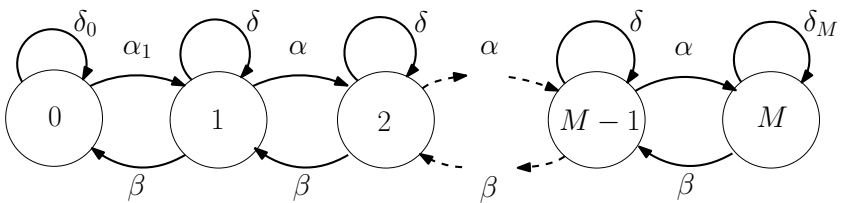

Figure 3: DTMC model for the common packet queue of length $M$ consisting of the generated CAM and DENM packets.

DENM packet trigger during $\tilde{T} \mathrm{~s}$ is given by $1-e^{-\lambda \tilde{T}}$. Due to its critical nature, a DENM packet is repeated $K$ times at a fixed period of $T_{D}$, for added reliability [24]. This means, the DENM generator captures two distinct packet types: a Poisson based triggered generation referred to as trigger and subsequent fixed-period repeat packet generations referred to as repetition. The trigger occurs only once per DENM event, and the repetition occurs $K-1$ times following a trigger, periodically, similar to CAM. Due to this reason, the CAM generation and the DENM repetition are modeled using the common states. Moreover, according to the standard [5], the originator vehicle has the liberty of setting $T_{D}$.

The packet generation is represented using states $(i, 0)$, $i \in\left\{t x, t x^{\prime}\right\}$. $t x$ and $t x^{\prime}$ is used to differentiate between the transmit status of the previously generated packet, i.e., whether it has been transmitted, or not, respectively. A packet generation is followed by a wait of $T_{l} \mathrm{~ms}, l \in\{C, D\}$, until the next packet generation. The waiting time is represented by states $(i, j), i \in\left\{t x, t x^{\prime}\right\}$ and $j \in\left[0, T_{l}-1\right]$, with a modeling granularity of $1 \mathrm{~ms}$ for $\mathrm{C}-\mathrm{V} 2 \mathrm{X}$ Mode 4 , referred to as a subframe, and $13 \mu$ s for IEEE $802.11 \mathrm{p}$, referred to as aSlotTime. If a transmission opportunity is not available upon generation, it waits for an opportunity, represented by states $\left(t x^{\prime}, j\right)$, $j \in\left[1, T_{l}-1\right]$. A successful transmission results in a state transition from $\left(t x^{\prime}, j\right)$ to $(t x, j-1), j \in\left[1, T_{l}-1\right] . P_{t}^{v 2 x}$ and $P_{t}^{11 p}$ denote the probability of transmitting a packet in C-V2X Mode 4 and IEEE 802.11 p, respectively, and these probability values link the generators with the DTMCs modeling the MAC layer operations as illustrated in Fig. 1.

Fig. 3 illustrates the DTMC that models the device level packet queue per vehicle, consisting of the generated CAM and DENM packets. State $(i), i \in[0, M]$, represents a queue length of $i$, where $M$ is the maximum length of the queue. It is not hard to see that the state transitions of this DTMC depend on the packet generation and transmission. Thus, the transition probabilities of the queue model are directly related to the packet generators, as shown in Fig. 1. A transmission of a packet may either lead to maintaining the current state $(i)$ or a state transition from $(i)$ to $(i-1)$, for $i \in[1, M]$, depending on whether a new packet has been generated concurrently, or not, respectively. Similarly, not being able to transmit a generated packet (i.e., traversing through states $\left(t x^{\prime}, j\right), j \in\left[0, T_{l}-1\right]$, in Fig. 2 without a transmission opportunity), leads to a state transition from $(i)$ to $(i+1)$, for $i \in[0, M-1]$. Let $P_{q e}$ denote the probability of the queue being empty, $P_{q n e}=1-P_{q e}$, and $P_{\text {arr }}$ denote the conditional probability of a new packet arrival given the queue is empty. $P_{q e}$ and $P_{a r r}$ link the queue model and the packet generators with the DTMCs modeling the MAC layer operation, respectively, as illustrated in Fig. 1. 


\section{B. DTMC model for $C-V 2 X$ Mode 4}

We begin this subsection by presenting the sensing-based SPS algorithm, which is used for radio resource allocation in C-V2X Mode 4. We follow it up with the respective DTMC model.

1) Semi-persistent scheduling algorithm: The SPS algorithm enables a vehicle to select radio resources without the assistance of an eNodeB, and each vehicle follows the following three steps for resource reservation.

Step 1: Within the selection window, which is the time window that initiates with a generation of a packet, vehicle $v$ identifies all possible candidate single-subframe resources (CSRs) that can be reserved. CSRs are groups of adjacent sub-channels within the given $1 \mathrm{~ms}$ subframe that are large enough to fit in the sidelink control information (SCI) and the transport block (TB) to be transmitted. The length of the selection window, which is denoted by $\Gamma$, is defined in the standard as the maximum latency in ms [9], and a CSR should be selected within this duration.

Step 2: Based on the information received in the previous 1000 subframes (sensing window), vehicle $v$ creates list $L_{1}$ that consists of CSRs that it can reserve. $L_{1}$ includes all the CSRs in the selection window except the ones that satisfy the following conditions.

1) CSRs used by vehicle $v$ during the sensing window. This is done as a precautionary measure due to vehicle $v$ not being able to sense these CSRs during its half-duplex transmissions.

2) CSRs that are being used by other vehicles at the time vehicle $v$ tries to utilize them (which are known thanks to the information contained in the SCI), and have a received signal strength indicator (RSSI) value above a threshold level $l_{t h}$.

If $L_{1}$ contains more than $20 \%$ of the total CSRs identified in Step 1 , the system moves to Step 3. Otherwise, $l_{t h}$ is increased by $3 \mathrm{~dB}$ and Step 2 is repeated.

Step 3: From $L_{1}$, vehicle $v$ filters out the CSRs that experience the lowest average RSSI values, where the averaging is done over the previous 10 subframes. These CSRs are added to a new list $L_{2}$ such that the size of $L_{2}$ amounts to $20 \%$ of the total CSRs in the selection window. Vehicle $v$ randomly and uniformly selects a CSR in $L_{2}$ and reserves it for the next $R C$ transmissions, where $R C$ denotes the value of the resource counter. Let $R C_{F} \in\left[R_{l}, R_{h}\right]$ denote the starting value of the resource counter, where $R_{h}$ and $R_{l}$ are upper and lower limits of $R C_{F}$, respectively. $R C$ is decremented by 1 for each transmission of a packet, which happens periodically every $\Gamma$ ms until $R C$ reaches 1 . When $R C=1$, new CSRs should be selected and reserved with probability $\left(1-P_{r k}\right)$, where $P_{r k} \in[0,0.8]$. This can be done by generating a number randomly and uniformly in $(0,1)$, and then comparing it with the predefined value of $P_{r k}$. Vehicle $v$ continues using the same CSR if the generated random number is less than $P_{r k}$, and it continues using the subframes encountered in intervals of $\Gamma \mathrm{ms}$ for the subsequent transmissions. Else, vehicle $v$ selects a new CSR for the next transmission from $L_{2}$. Upon new CSR selection, the vehicle randomly and uniformly

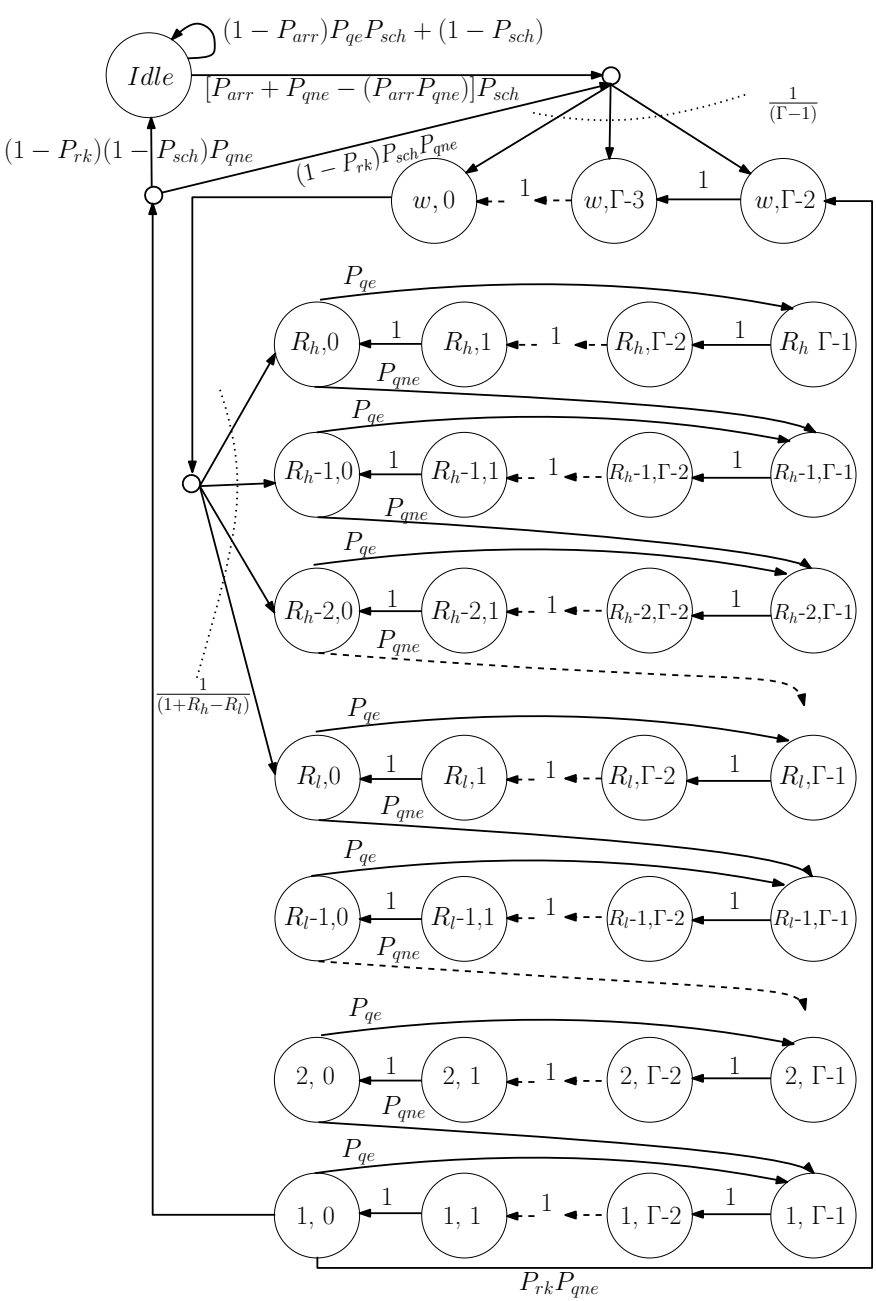

Figure 4: The DTMC modeling the MAC layer operations of C-V2X Mode 4.

selects a subframe that falls within the next $\Gamma \mathrm{ms}$ for the next transmission. Please refer to [20] for a pseudo-code of this algorithm.

2) DTMC Model: Fig. 4 illustrates the DTMC model for C-V2X Mode 4 operation. The state-space of the model is denoted by $S^{v 2 x}$. Let $P_{\text {sch }}$ denote the probability of allocating a suitable CSR for a vehicle through Steps 1-3. State (Idle) represents the state with no packets to transmit, or no CSRs to transmit. According to the standard [8], there are three selection window sizes with respective ranges for $R C_{F}$. To this end, the standard includes $\Gamma=100 \mathrm{~ms}$ with $R C_{F} \in[5,15], \Gamma=50$ ms with $R C_{F} \in[10,30]$ and $\Gamma=20 \mathrm{~ms}$ with $R C_{F} \in[25,75]$.

Consider the arrival of a new packet while vehicle $v$ is idle. This necessitates the allocation of a CSR utilizing the SPS algorithm. As $\Gamma \mathrm{ms}$ is the maximum allowable latency, the transmission should happen within the next $\Gamma$ subframes. Upon allocation of the CSR, vehicle $v$ selects (randomly and uniformly) a subframe for transmission. Thus, the waiting time before the transmission is modeled by assuming $\Gamma-1$ equiprobable states $(w, j)$, where $j \in[0, \Gamma-2]$. As the waiting time elapses, vehicle $v$ selects a value for $R C$ randomly and uniformly from the set of $\left(1+R_{h}-R_{l}\right)$ values. At every state $(i, 0)$, where $i \in\left[1, R_{h}\right]$, there is a transmission opportunity, and $i$ represents the current $R C$ value. The device utilizes 
this opportunity to transmit the control information related to its persistent scheduling. If the queue is not empty, the transmission opportunity is also utilized for data transmission, $i$ is decremented, and the vehicle waits for the next transmission opportunity that arises in $\Gamma \mathrm{ms}$. This waiting time is represented by states $(i-1, j)$, where $j \in[1, \Gamma-1]$. On the other hand, if the queue is empty, the vehicle similarly waits $\Gamma$ $\mathrm{ms}$ for the next transmission opportunity. We consider that the vehicle maintains the same $R C$ value $i$ during this waiting period $^{1}$. This process repeats until the system reaches state $(1,0)$.

If the queue is still not empty at state $(1,0)$, the vehicle has the option of using the same CSR (with probability $P_{r k}$ ), or choose a new CSR. If the same CSR is used, the vehicle waits for the maximum waiting time of $\Gamma-1 \mathrm{~ms}$ before choosing a $R C$ value and transmitting. The state transitions for selecting a new radio resource are similar to the transitions described for packet arrival while vehicle $v$ being idle. It is not hard to see that selecting a new radio resource may lead to a lower delay due to the possibility of a lower waiting time.

\section{DTMC model for IEEE 802.11p}

The state space of the model is denoted by $S^{11 p}$ and the DTMC model is presented in Fig. 5. $\check{C}$ denotes the minimum contention window size. State (Idle) represents the state where there are no packet arrivals, thus the queue is empty. If a packet arrives while being idle, the MAC protocol listens for an $A I F S$ duration before transmitting. The $A I F S$ duration is calculated according to AIFS = aSIFSTime $+A I F S N *$ aSlotTime, where aSIFSTime is $32 \mu \mathrm{s}$, and the AIFSN value is selected according to the access category (AC). ETSI specifications do provide four ACs: background, best effort, video, and voice. In this paper, we assume that both CAM and DENM packets utilize the best effort AC. We thus have $A I F S N=6$ and $\check{C}=15$ according to the standard [6].

States $\left(A_{i}\right)$ for $i \in\{1, \ldots, \Omega\}$, represent the $A I F S$ waiting time, and $\Omega$ denotes the maximum number of aSlotTime intervals per $A I F S$ duration. $\theta$ represents the probability of the channel being busy (channel busy ratio). If the channel is found idle for an $A I F S$ duration, the vehicle is allowed to transmit. Data transmission is represented by states $(T x, i)$, where $i \in\{1, \ldots, \vartheta\}$, and $\vartheta$ denotes the number of aSlotTime intervals required to transmit a packet of 134 bytes over a $6 \mathrm{Mbps}$ control channel $(\mathrm{CCH})$ [7].

If the channel becomes busy during the AIFS duration, the vehicle waits for $\vartheta \times a$ SlotTime, which is the time taken for data transmission, until the channel is free again. Waiting is represented by states $(B, i)$, where $i \in\{1, \ldots, \vartheta\}$. The channel being busy at state $A_{1}$ depicts a scenario where the packet arrival of the vehicle of interest has occurred while the channel is busy, i.e., another vehicle is transmitting. Thus, the time it has to wait before sensing a free channel is given by

\footnotetext{
${ }^{1}$ As found often in standardization, the standard does not specifically describe what needs to happen to the $R C$ value in such a scenario. Any realization that fulfills the requirements of the standard is deemed to be correct. Note that both decrementing the $R C$ value or maintaining the same $R C$ value during this waiting period satisfy the standard. We have used the latter for our model.
}

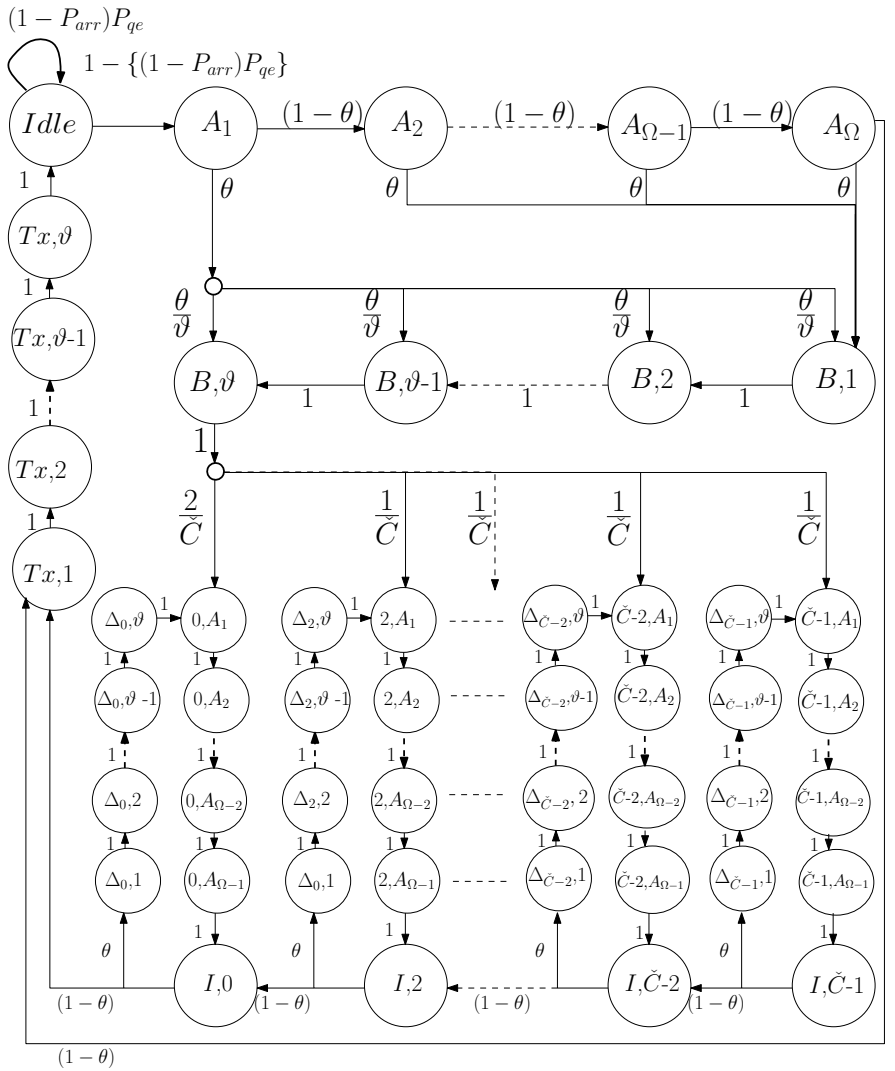

Figure 5: The DTMC modeling the MAC layer operations of IEEE 802.11p.

$\varrho \times$ aSlotTime, where $\varrho$ is a uniformly distributed random integer in $[1, \vartheta]$.

When the channel becomes free again, that is at state $(B, \vartheta)$, vehicle $v$ initiates a backoff process. The backoff counter value is selected randomly (uniformly) in $[0, \check{C}]$, and the backoff stage is selected depending on the respective backoff counter value. According to the standard [6], backoff counter value 0 and 1 both lead to backoff stage 0 . Thus, the probability of selecting backoff stage 0 is twice the probability of selecting any other backoff stage. Vehicle $v$ waits for another $A I F S$ duration before sensing the channel again. For backoff counter value $i \in\{0, \ldots,(\check{C}-1)\}$, states $\left(i, A_{j}\right)$, where $j \in\{1, \ldots,(\Omega-1)\}$, represent the waiting duration, and $(I, i)$ represent the sensing states. If the channel is found busy at state $(I, i)$, vehicle $v$ waits for $\vartheta \times a$ SlotTime, which is represented by states $\left(\Delta_{i}, j\right)$, where $j \in\{1, \ldots, \vartheta\}$, and another $A I F S$ duration at the same backoff stage $i$. This loop continues until the channel is found idle at state $(I, i)$. The backoff counter is decremented when the channel is found idle, which takes us to state $(I, i-1)$. If vehicle $v$ finds the channel to be free at state $(I, 0)$, it transmits data.

\section{Steady-STATE SOlutions}

Steady-state solutions of the DTMCs are presented in this section for a system with $N$ vehicles. Firstly, we focus on the CAM and DENM packet generators and the device level packet queue. By utilizing these results, we present the steadystate solutions of the DTMC models developed for C-V2X Mode 4 and IEEE 802.11p MAC layer operations. 


\section{A. Queue Model}

We have already discussed the importance of $P_{q e}$, as it links the DTMCs modeling the MAC layer operation with the DTMC modeling the device level packet queue. This value can be obtained through the steady-state probability of state $(0)$ in the queue model. From Fig. 3, the steady-sate probability of state $(0)$ can be written as

$$
\pi_{0}=\left[1+\alpha_{1}\left(\frac{1-\beta^{-M} \alpha^{M}}{\beta-\alpha}\right)\right]^{-1}=P_{q e} .
$$

To obtain $\alpha, \alpha_{1}$, and $\beta$, we need the steady-state solutions of the CAM and DENM generators, as shown in Fig. 1.

Let $\pi_{i, j}^{C}$ and $\pi_{i, j}^{D}$ denote the steady-state probabilities of state $(i, j)$ of the CAM and the DENM generators, respectively. To this end, the steady-state probabilities of the CAM generator are given by

$\pi_{t x, 0}^{C}=\left[1-\left(1-P_{t}^{k}\right)^{T_{C}-1}\right]\left[T_{C}\left[1-P_{t}^{k}\left(1-P_{t}^{k}\right)^{T_{C}-1}\right]\right]^{-1}$, $\pi_{t x, j}^{C}=P_{t}^{k}\left(\pi_{t x, 0}^{C}+\sum_{l=j+1}^{T_{C}-1} \pi_{t x^{\prime}, l}^{C}\right)$ for $j \in\left[1, T_{C}-2\right]$, $\pi_{t x, T_{C}-1}^{C}=\pi_{t x, 0} P_{t}^{k}, \pi_{t x^{\prime}, j}^{C}=\pi_{t x, 0}^{C}\left[\left(1-P_{t}^{k}\right)^{T_{C}-j}\right][1-(1-$ $\left.\left.P_{t}^{k}\right)^{T_{C}-1}\right]^{-1}$ for $j \in\left[0, T_{C}-1\right]$.

Similarly, the steady-state probabilities of the DENM generator are given by

$\pi_{t x, 0}^{D}=\left[\left(1-\frac{1}{K}\right) \frac{T_{D}\left[1-P_{t}^{k}\left(1-P_{t}^{k}\right)^{T_{D}-1}\right]}{1-\left(1-P_{t}^{k}\right)^{T_{D}-1}}+\frac{1}{K}+\frac{1}{K\left(1-e^{-\lambda \widetilde{T}}\right)}\right]^{-1}$, $\pi_{t x, j}^{D}=P_{t}^{k}\left[\left(1-\frac{1}{K}\right) \pi_{t x, 0}^{D}+\sum_{l=j+1}^{T_{D}-1} \pi_{t x^{\prime}, l}^{D}\right]$ for $j \in\left[1, T_{D}-2\right]$, $\pi_{t x, j}^{D}=\pi_{t x, 0}^{D}\left(1-\frac{1}{K}\right) P_{t}^{k}$ for $j=T_{D}-1, \pi_{t x^{\prime}, j}^{D}=\pi_{t x, 0}^{D}(1-$ $\left.\frac{1}{K}\right)\left[\left(1-P_{t}^{k}\right)^{T_{D}-j}\right]\left[1-\left(1-P_{t}^{k}\right)^{T_{D}-1}\right]^{-1}$ for $j \in\left[0, T_{D}-1\right]$.

Since we are using a single queue for both CAM and DENM packets, the transition probabilities of the queue model in Fig. 3 depend on both generator models that run simultaneously. For $x \in\left\{\alpha, \alpha_{1}, \beta\right\}$, let $x^{C}$ and $x^{D}$ denote the resulting transition probability if only the CAM generator or the DENM generator is in operation, respectively. For two events $E_{1}$ and $E_{2}, \operatorname{Pr}\left\{E_{1} \cup E_{2}\right\}=\operatorname{Pr}\left\{E_{1}\right\}+\operatorname{Pr}\left\{E_{2}\right\}-\operatorname{Pr}\left\{E_{1} \cap E_{2}\right\}$. Thus, $x=x^{C}+x^{D}-x^{C} x^{D}$ for $x \in\left\{\alpha, \alpha_{1}, \beta\right\}$. To this end, $\alpha^{C}=\pi_{t x^{\prime}, 0}^{C}, \quad \alpha_{1}^{C}=\pi_{t x, 0}^{C}\left(1-P_{t}^{k}\right), \beta^{C}=\sum_{j=1}^{T_{C}-1} \pi_{t x^{\prime}, j}^{C} P_{t}^{k}$, $\alpha^{D}=\pi_{t x^{\prime}, 0}^{D}, \quad \alpha_{1}^{D}=\pi_{t x, 0}^{D}\left(1-\frac{1}{K}\right)\left(1-P_{t}^{k}\right)$, and $\beta^{D}=$ $\sum_{j=1}^{T_{D}-1} \pi_{t x^{\prime}, j}^{D} P_{t}^{k}$, for $k \in\{v 2 x, 11 p\}$. With similar reasoning, $P_{\text {arr }}=\pi_{t x, 0}^{C}+1-e^{-\lambda \tilde{T}}-\pi_{t x, 0}^{C}\left(1-e^{-\lambda \tilde{T}}\right)$.

\section{B. DTMC model for $C-V 2 X$}

According to the 3GPP C-V2X standard [9], single-carrier frequency-division multiple access (SC-FDMA) is considered for the uplink, using a $10 \mathrm{MHz}$ channel. 50 resource blocks (RB) are allocated for this bandwidth per each slot (half subframe), and hence, one subframe contains 100 RBs. A CSR requires at least $4 \mathrm{RBs}$ to transmit a 100 byte payload, using 64 QAM modulation. Therefore, each $1 \mathrm{~ms}$ subframe can hold up to 25 CSRs, and hence, the largest selection window of 100 ms can hold up to 2500 CSRs. $20 \%$ of this is 500 , and $l_{t h}$ can be fine-tuned until we end up with the required number of CSRs. Thus, the standard itself makes it highly unlikely that a randomly selected vehicle ends up without an allocated CSR. Thus, we consider $P_{\text {sch }}=1$ in our study without any loss of generality. Now that we have obtained $P_{q e}$ and $P_{q n e}$, the steady-state solutions of the DTMC model for C-V2X can be used to obtain $P_{t}^{v 2 x}$ found in the packet generators.

The steady-state equations of the DTMC model in Fig. 4 are used to derive expressions for its steady-state probabilities, which are presented next. To this end,

State (Idle):

$$
\pi_{I d l e}^{v 2 x}=b \pi_{w, 0}, \text { where } b=\frac{\left(1-P_{r k}\right)\left(\frac{1}{P_{s c h}}-1\right)}{P_{\text {arr }}+P_{q n e}\left(1-P_{a r r}\right)} .
$$

States $(w, j)$ : for $j \in[0, \Gamma-2]$

$$
\begin{aligned}
& \pi_{w, j}=\left[1-\frac{j}{(\Gamma-1)}\right]\left[a \pi_{\text {Idle }}^{v 2 x}+\left(1-P_{r k}\right) P_{s c h} \pi_{1,0} P_{q n e}\right]+ \\
& P_{r k} \pi_{1,0} P_{q n e},
\end{aligned}
$$

where $a=\left(P_{a r r}+P_{q n e}-P_{a r r} P_{q n e}\right) P_{s c h}$.

States $(i, j)$ :

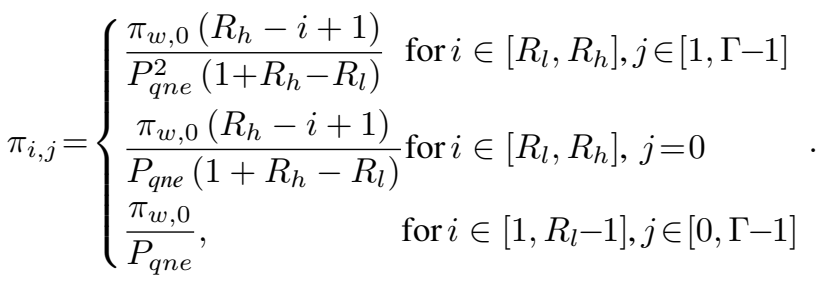

Since the sum of these steady-state probabilities is one, we get

$$
\begin{aligned}
& \pi_{w, 0}=\left[1-\Gamma+b+\left(\frac{\Gamma-2}{2}\right)\left[a b+2 P_{r k}+\left(1-P_{r k}\right) P_{s c h}\right]+\right. \\
& \left.\frac{\Gamma\left(R_{h}+R_{l}\right)}{2 P_{\text {qne }}}\right]^{-1}
\end{aligned}
$$

The probability of transmission opportunity can be written as $P_{t x o}=\sum_{i=1}^{R_{h}} \pi_{i, 0}$, for which an expression can be obtained by substituting (3) in (2). Finally, we find $P_{t}^{v 2 x}$ through the product of $P_{t x o}$ and $P_{q n e}$.

\section{DTMC model for ETSI ITS-G5 based IEEE 802.11p}

Next, we present the steady-state solutions for the DTMC model in Fig. 5. To this end,

States $\left(A_{i}\right)$ : for $i \in[1, \Omega]$

$$
\pi_{A_{i}}=\pi_{\text {Idle }}^{11 p}\left[1-P_{q e}\left(1-P_{\text {arr }}\right)\right](1-\theta)^{(i-1)} .
$$

States $(B, j)$ : for $j \in[1, \vartheta]$

$$
\pi_{B, j}=\pi_{\text {Idle }}^{11 p}\left[1-P_{q e}\left(1-P_{\text {arr }}\right)\right]\left[\frac{\theta}{\vartheta} j-(1-\theta)^{\Omega}-\theta+1\right] .
$$

States $\left(\Delta_{i}, j\right):$ for $i \in[0,(\check{C}-1)], j \in[1, \vartheta]$

$$
\pi_{\Delta_{i}, j}=\pi_{B, \vartheta} \frac{(\check{C}-i) \theta}{\check{C}(1-\theta)} .
$$

States $\left(i, A_{j}\right)$ :

$\pi_{i, A_{j}}=\left\{\begin{array}{l}\frac{\pi_{B, \vartheta}[1+(\check{C}-i-1) \theta]}{\check{C}(1-\theta)} \text { for } i \in[2,(\check{C}-1)], j \in[1,(\Omega-1)] \\ \frac{\pi_{B, \vartheta}(2-2 \theta+\check{C} \theta)}{\check{C}(1-\theta)} \text { for } i=0, j \in[1,(\Omega-1)]\end{array}\right.$. 


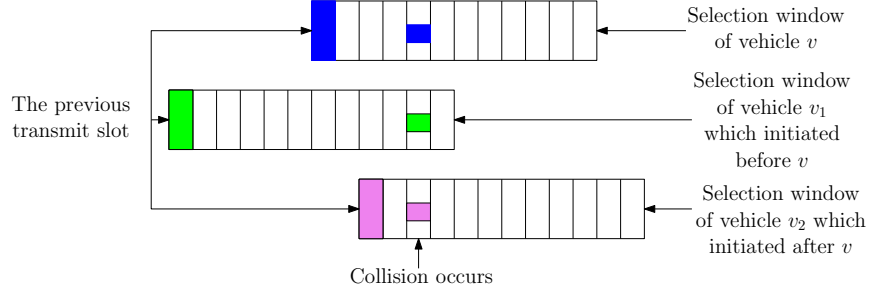

Figure 6: An illustration of a possible collision in C-V2X Mode 4

States $(I, j)$ : for $j \in[0,(\check{C}-1)] \pi_{I, j}=\pi_{B, \vartheta} \frac{(\check{C}-j)}{\check{C}(1-\theta)}$.

States $(T x, j)$ : for $j \in[1, \vartheta] \pi_{T x, j}=\pi_{\text {Idle }}^{11 p}\left[1-P_{q e}\left(1-P_{\text {arr }}\right)\right]$.

Since the sum of these steady-state probabilities is one, we get

$$
\begin{aligned}
& \pi_{\text {Idle }}^{11 p}=\left[1+\left[1-\left(1-P_{\text {arr }}\right) P_{q e}\right]\left[[ 1 - ( 1 - \theta ) ^ { \Omega } ] \left[\frac{1}{\theta}+\frac{(\check{C}+1) \theta \vartheta}{2(1-\theta)}\right.\right.\right. \\
& +\vartheta+(\Omega-1) \frac{[(\check{C}-2)[(\check{C}-3) \theta+2]+(4-4 \theta+2 \check{C} \theta)]}{2 \check{C}(1-\theta)} \\
& \left.\left.\left.+\frac{(\check{C}+1)}{2(1-\theta)}\right]+\frac{\theta}{2}(1-\vartheta)+\vartheta\right]\right]^{-1}
\end{aligned}
$$

The obtained solution for $\pi_{\text {Idle }}^{11 p}$ is substituted in (4) to determine $P_{t}^{11 p}=\sum_{j=1}^{\vartheta} \pi_{T x, j}$ and $\theta=1-\left(1-\sum_{j=1}^{\vartheta} \pi_{T x, j}\right)^{(N-1)}$.

\section{Performance Analysis}

This section focuses on deriving expressions for several useful performance parameters that can be used to compare the MAC layer performance of C-V2X Mode 4 and IEEE $802.11 \mathrm{p}$.

\section{A. Probability of Collision $P_{c o l}$}

Even though the SPS algorithm attempts to minimize packet collisions between vehicles at transmission by considering the radio resource utilization of vehicles during the $1000 \mathrm{~ms}$ sensing window, there still remains a possibility for collisions. To this end, a schedule collision can occur when a vehicle selects a new radio resource for transmission. In particular, a collision can occur when there is an overlap in the selection windows of neighboring vehicles, as illustrated in Fig. 6. In such a scenario, the vehicles with overlap select a CSR independent of each other, and hence, there is a possibility of them selecting the same CSR that leads to collision. Let $C S R_{\text {tot }}$ denote the total number of CSRs in the selection window. We start the analysis by obtaining an expression for the collision probability of $\mathrm{C}-\mathrm{V} 2 \mathrm{X}$ Mode 4.

Lemma 1: The collision probability of $\mathrm{C}-\mathrm{V} 2 \mathrm{X}$ Mode 4 is given by

$P_{c o l}^{v 2 x} \approx 1-\left[1-\left[1-\prod_{i=0}^{\Gamma-1}\left(1-\frac{\pi_{i, 0}}{1-\pi_{i, 0} i}\right)\right] \frac{\left(1-P_{r k}\right)}{\left(C S R_{t o t}-N+1\right)}\right]^{N-1}$

Proof: See Appendix A.

The collision probability of IEEE $802.11 \mathrm{p}$ is calculated according to $P_{c o l}^{11 p}=1-P_{s u c}$, where $P_{\text {suc }}$ is the conditional probability that exactly one vehicle transmits on the channel, given that at least one vehicle transmits [29]. An expression for the collision probability of IEEE $802.11 \mathrm{p}$ is formally stated through the following lemma.

Lemma 2: The collision probability of IEEE $802.11 \mathrm{p}$ is given by

$$
\frac{N\left[(1-\theta)\left(\pi_{I, 0}+\pi_{A_{\Omega}}\right)+\sum_{j=1}^{\vartheta} \pi_{T x, j}\right]\left[1-\left(\pi_{I, 0}+\pi_{A_{\Omega}}+\sum_{j=1}^{\vartheta} \pi_{T x, j}\right)\right]^{(N-1)}}{1-\left[1-\left(\pi_{I, 0}+\pi_{A_{\Omega}}+\sum_{j=1}^{\vartheta} \pi_{T x, j}\right)\right]^{N}} .
$$

Proof: See Appendix A.

\section{B. Average Delay $D_{\text {ave }}$}

Next, we focus on the average delay between the generation and the transmission of a packet. The delay value captures the queuing delay, which is the time a packet waits in the queue making way to the previously generated packets, and the access delay, which is the time a vehicle waits before being able to access the radio resources. Firstly, we present an expression for the average delay of C-V2X Mode 4 through the following lemma.

Lemma 3: The average delay of C-V2X Mode 4 is given by

$$
d_{a v g}^{v 2 x}=\frac{\sum_{i=1}^{M} \frac{2 i-1}{2 P_{t x o}} \pi_{i}}{1-P_{q e}} .
$$

Proof: See Appendix B.

For IEEE $802.11 \mathrm{p}$, the average delay is calculated by utilizing the delay of each state in the DTMC model, except the idle state. The normalized average delay of the system is calculated using the delay values of the individual states. Let $D_{i, j}$ denote the delay at state $(i, j)$. aSlotTime is used as the unit delay, thus $D_{T, 1}=\vartheta$ since the transmission of a packet of 134 bytes takes $\vartheta \times$ aSlotTimes. We assume $D_{I}=0$. To this end, the delay at each state of the system is calculated according to the following equations.

States $(I, j)$ :

$D_{I, j}=\left\{\begin{array}{ll}\frac{1+\vartheta+\theta(\Omega-1)}{(1-\theta)} & \text { for } j=0 \\ \frac{j+\vartheta[1+\theta(j-1)]+j \theta(\Omega-1)}{(1-\theta)} & \text { for } j \in[2, \check{C}-1]\end{array}\right.$.

States $\left(i, A_{j}\right)$ : for $i \in\{0,2, \ldots, \check{C}-1\}, j \in[1,(\Omega-1)]$

$$
D_{i, A_{j}}=(\Omega-j)+D_{I, j} .
$$

State $\left(\Delta_{i}, j\right)$ : for $i \in\{0,2, \ldots, \check{C}-1\}, j \in[1, \vartheta]$

$$
D_{\Delta_{i}, j}=(\vartheta-j+1)+(\Omega-1)+D_{I, j} .
$$

States $(B, j)$ :

$$
D_{B, j}=\left\{\begin{array}{rl}
1+\frac{2}{\check{C}}\left[(\Omega-1)+D_{I, 0}\right]+\frac{(\check{C}-2)(\Omega-1)}{\check{C}}+\sum_{j=2}^{\check{C}-1} D_{I, j} \\
\text { for } j=\vartheta \\
1+D_{B,(j+1)} \text { for } j \in[1,(\vartheta-1)]
\end{array} .\right.
$$


States $(T x, j)$ : for $j \in[1, \vartheta] D_{T x, j}=\vartheta-(j-1)$.

States $\left(A_{i}\right)$ :

$$
D_{A_{i}}= \begin{cases}1+(1-\theta) D_{A_{i+1}}+\frac{\theta}{\vartheta} \sum_{j=1}^{\vartheta} D_{B, j} & \text { for } i=1 \\ 1+(1-\theta) D_{A_{i+1}}+\theta D_{B, 1} & \text { for } i \in[2,(\Omega-1)] \\ 1+(1-\theta) D_{T x, 1}+\theta D_{B, 1} & \text { for } i=\Omega\end{cases}
$$

By using these equations, the average delay of the system is obtained through the following lemma.

Lemma 4: The average delay of IEEE $802.11 \mathrm{p}$ is given by

$$
\begin{aligned}
& d_{\text {avg }}^{11 p}=\vartheta+1+\sum_{i=1}^{\Omega-1}(1-\theta)^{i}+ \\
& \sum_{i \in \mathcal{S}} \frac{D_{i} \pi_{i}}{\left[1-\left(\pi_{\text {Idle }}^{11 p}+\sum_{j=1}^{\vartheta} \pi_{T x, j}+\sum_{k=1}^{\Omega} \pi_{A_{k}}\right)\right]},
\end{aligned}
$$

where $\mathcal{S}=\left\{S^{11 p}-\left\{I d l e, \bigcup_{j=1}^{\vartheta}(T x, j), \bigcup_{k=1}^{\Omega} A_{k}\right\}\right\}$.

Proof: See Appendix B.

\section{Average Channel Utilization}

The average channel utilization depicts the average number of users successfully accessing the channel simultaneously. Thus, the average channel utilization of C-V2X Mode 4 and IEEE $802.11 \mathrm{p}$ is given by

$$
C U_{\text {avg }}^{v 2 x}=\frac{P_{t}^{v 2 x} N\left(1-P_{c o l}^{v 2 x}\right)}{C S R s \text { per subframe }}
$$

and

$$
C U_{a v g}^{11 p}=P_{t}^{11 p} N\left(1-P_{c o l}^{11 p}\right)
$$

respectively. Note that since we are interested in finding the average channel utilization within a single subframe in C-V2X Mode 4, we normalize the channel utilization value by the total number of CSRs within a single subframe, which is given by $C S R_{t o t} / \Gamma$.

\section{Numerical Results and Discussion}

In this section, we present an application of the models to provide insights and comparisons on key performance indicators through numerical evaluations. We were unable to find or generate similar data from a real vehicular network testbed for validation.

\section{A. Instantiation of CAM, DENM and the DTMC Models in a Highway}

We consider a highway with four parallel lanes in each direction, with an average inter-vehicle gap of $50 \mathrm{~m}$. Note that we have not done any location modeling, mobility modeling, or PHY-layer signal modeling. Thus, the highway scenario is only used as an illustrative setting such that it provides a feasible range for $N$ within the coverage region of vehicle $v$. We also assume that only CAM and DENM are utilized for V2V communication [30], [31], while their reference packet formats are specified according to ETSI [4], [5]. We consider
$T_{C}$ to be between $100 \mathrm{~ms}$ and $1 \mathrm{~s}$ [4], and $T_{D}$ to be 100,200 and $300 \mathrm{~ms}$ [5]. $K$ is set at three arbitrary values 2,5 , and 9 to study scenarios with low, medium, and high repetitions, respectively. The standard allows the vehicle to select $T_{D}$ and $K$ based on the severity of the event. Two candidate values for $\lambda$ are selected to study low and high packet generation rates, and the values are set by taking the use case scenarios in [32] into consideration. $M$ is set at 10 in the queue model. In IEEE $802.11 \mathrm{p}, T_{C}$ is regulated under the transmit rate control (TRC) technique of ETSI ITS-G5 decentralized congestion control (DCC) algorithm, where during periods of high/low utilization, $T_{C}$ is increased/decreased to manage congestion. This is termed as an adaptive CAM rate in the numerical results. The steady-state probabilities of the DTMCs are calculated in parallel, which are then used to calculate the probability values that link the DTMCs, as shown in Fig. 1. The probability values are iteratively recomputed until they converge.

1) Average delay: Average delay is calculated according to (8) and (9) for C-V2X Mode 4 and IEEE 802.11p, respectively. Fig. 7a illustrates the variation of the average delay with $N$. Firstly, we can observe that IEEE $802.11 \mathrm{p}$ is superior to $\mathrm{C}-\mathrm{V} 2 \mathrm{X}$ Mode 4 in terms of the average delay. The lower delay in IEEE $802.11 \mathrm{p}$ is mainly due to the maximum $A I F S$ duration being $149 \mu$ s [6]. This is approximately equal to 12 aSlotTimes, thus to transmit a 134 byte packet, it takes 14 aSlotTimes over the $\mathrm{CCH}$. Therefore, even after adding the average backoff delay to the above-calculated delay, it is unlikely that the total average delay is greater than a few milliseconds. This is much smaller compared $20 \mathrm{~ms}$, which is the smallest selection window size in C-V2X Mode 4, and where it does the best in terms of average delay as shown in Fig. 7a. The observation implies that IEEE $802.11 \mathrm{p}$ is a better choice for delay-critical use case scenarios such as emergency electronic brake lights, emergency vehicle warning, vulnerable road user warning, and pre-crash sensing warning.

According to Fig. 7a, we can also observe that the average delay increases further with $\Gamma$ in C-V2X Mode 4. Thus, $\Gamma$ should be set small for low-latency applications. However, reducing $\Gamma$ in turn reduces the number of CSR values, and hence, it reduces the number of vehicles that can be supported simultaneously, introducing a tradeoff. Thus, reducing $\Gamma$ is more suited for a sparsely-populated vehicular network. For a given value of $\Gamma$, a higher average delay can be observed when the CAM inter-arrival time is reduced from 200 to $100 \mathrm{~ms}$. This is mainly due to the increased congestion in the network.

It is interesting to note from Fig. 7a that the average delay is not sensitive to the number of vehicles in $\mathrm{C}-\mathrm{V} 2 \mathrm{X}$ Mode 4 compared to IEEE 802.11 p, where the average delay increases with $N$. C-V2X Mode 4 uses a scheduling based resource allocation method, and in this setting and the range considered for $N$, there exist ample radio resources for all users to transmit. This is the main reason for the flat behavior of the average delay with respect to $N$. On the other hand, IEEE 802.11p resorts to a contention-based access mechanism. Therefore, the delay increases monotonically with $N$. The explanation is consistent with Fig. $7 \mathrm{~b}$ that depicts the channelbusy ratio $\theta$, which is a metric used to capture the busyness of 


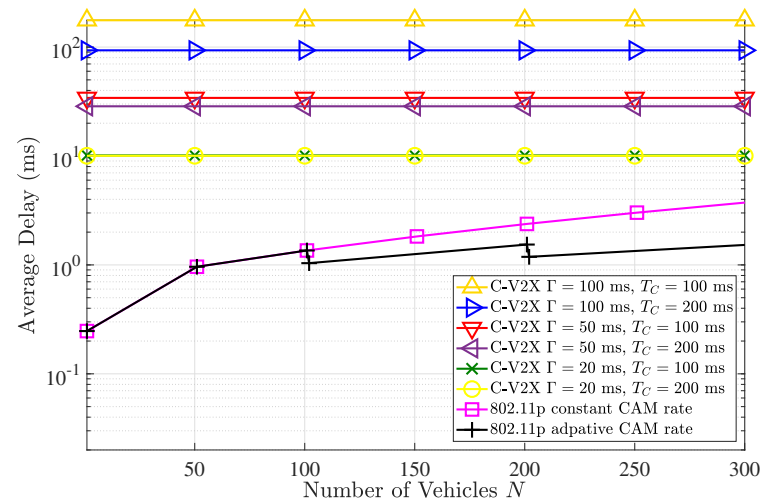

(a) The average delay vs $N$, where $P_{r k}=0.4$.

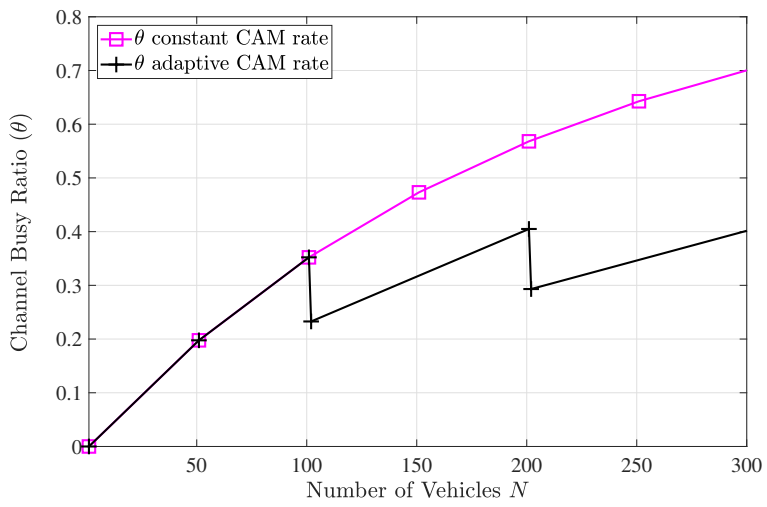

(b) The channel busy ratio vs $N$, where $T_{C}=100 \mathrm{~ms}$.

Figure 7: The behavior of the average delay and the channel busy ratio with $N$, where $\lambda=1, T_{D}=100 \mathrm{~ms}$, and $K=5$.

the channel in IEEE 802.11p. The channel is considered to be busy if a vehicle other than the target vehicle is transmitting. Due to the significant variation in the average delay of IEEE 802.11 p with $N$, there may be scenarios where C-V2X Mode 4 may be the better choice for delay critical applications in densely-populated vehicular networks. It can also be seen from Fig. $7 \mathrm{~b}$ that the adaptive CAM rate affects the channel-busy ratio favorably, and thus helps in reducing the average delay associated with IEEE 802.11p further, as can be observed in Fig. 7a. This implies that adaptive CAM facilitates a higher number of vehicles in a network that utilizes IEEE $802.11 \mathrm{p}$ for communication without violating the stringent delay constraints.

The average delay variation of both technologies with $T_{C}$ is shown in Fig. 8a. Intuitively, the behavior with $T_{D}$ should be similar as the modeling of CAM generation and DENM repetition is identical. Firstly, we can see the average delay reducing with $T_{C}$ as observed and explained in Fig. 7a. Secondly, with regards to $\mathrm{C}-\mathrm{V} 2 \mathrm{X}$, we can see that $\Gamma$ has a higher impact on the average delay than $T_{C}$. The value of $\Gamma$ dominates the delay, i.e., we cannot negate the adverse effect on the delay caused by an increase in $\Gamma$ by simultaneously increasing $T_{C}$. We note that there is a tradeoff in reducing $\Gamma$ as well. While reducing the average delay, it simultaneously reduces the number of CSR values, and hence, it reduces the number of vehicles that can be supported simultaneously. The average delay associated with IEEE 802.11 p reduces monotonically

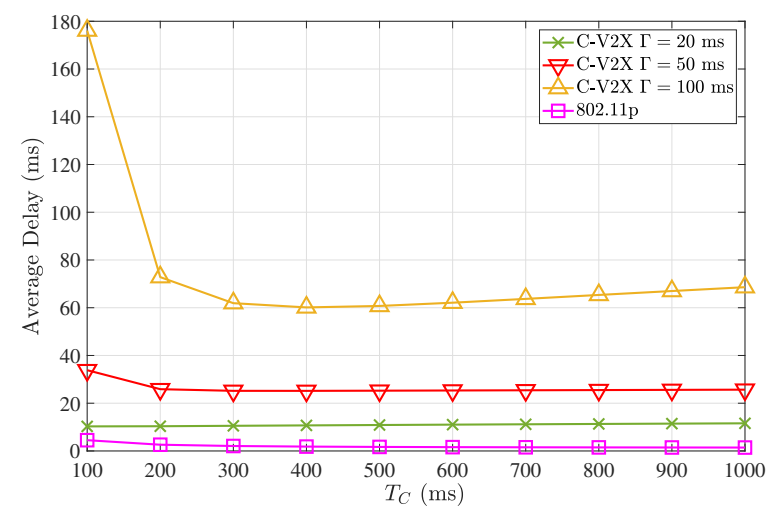

(a) Average delay vs $T_{C}, N=300, \lambda=1, T_{D}=100 \mathrm{~ms}, P_{r k}=0.4$, and $K=5$.

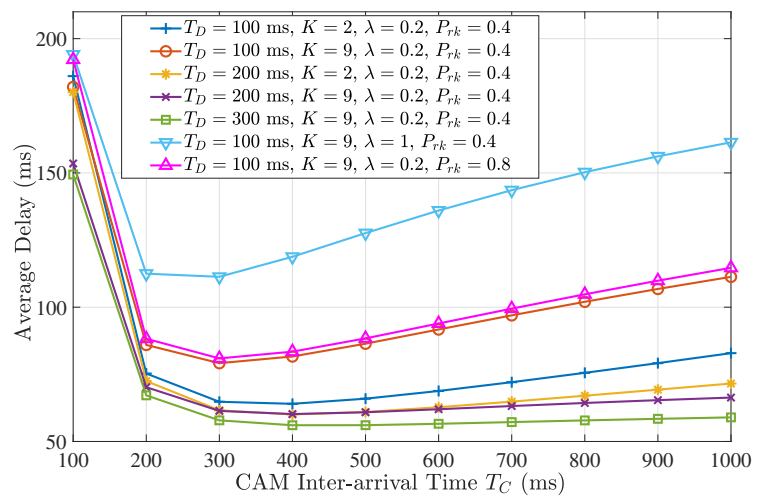

(b) Average delay of C-V2X Mode 4 vs $T_{C}$ for different combinations of $T_{D}, K, \lambda$ and $P_{r k}$, where $N=50$, and $\Gamma=100 \mathrm{~ms}$.

Figure 8: The behavior of the average delay with the inter-arrival time of CAM packets $T_{C}$.

with $T_{C}$. The corresponding behavior associated with C-V2X is more interesting as it first decreases and then increases with regards to $T_{C}$ (the variation is negligibly small for $\Gamma=20$ $\mathrm{ms})$. Thus, we further elaborate on the variation of the average delay of C-V2X Mode 4 with $T_{C}, P_{r k}$, and different parameter combinations of the DENM generator model such as $T_{D}$ and $K$ in Fig. 8b.

According to Fig. $8 \mathrm{~b}$, we can observe that the average delay increases when $T_{D}$ decreases or when the DENM packet arrival rate increases. Both of these observations are due to the congestion caused by more transmissions per unit time. When $T_{D}=100 \mathrm{~ms}$, the average delay increases with $K$. However, when $T_{D}=200 \mathrm{~ms}$, the average delay decreases with $K$. This phenomenon can be justified as follows. When $T_{D}=100$ $\mathrm{ms}$, the service rate is nearly equal to the packet repetition frequency. This results in more CAM and DENM packets in the queue, leading to higher queuing delays. However, when $T_{D}=200 \mathrm{~ms}$, the service rate is higher than the repetition interval of DENM packets. In such a scenario, increasing the average number of repetitions results in the target vehicle encountering the random waiting time, which has a maximum average delay of $50 \mathrm{~ms}$ (when $\Gamma=100 \mathrm{~ms}$ ), more frequently compared to waiting through the whole resource reservation interval (RRI), which is of $100 \mathrm{~ms}$. This leads to a reduction in the average delay. Similar behavior can be observed for $T_{D}=300 \mathrm{~ms}$ as well.

An interesting observation in the behavior of the average 
delay is the existence of a locally optimal point. For example, when $T_{D}=100 \mathrm{~ms}, K=9$ and $\lambda=0.2$ packets $/ \mathrm{s}$, the lowest average delay can be observed at $T_{C}=300 \mathrm{~ms}$. This implies that the average delay can be reduced further by dynamically changing the CAM packet generation rate based on the generation rate of DENM packets. The DENM packet generation rate is based on the occurrence of an event and its severity. It can be concluded that the vehicle can reduce the overall average delay further in C-V2X Mode 4 communication if it can change the CAM packet generation rate based on $\lambda$, to achieve the local optimal point of the delay curve shown in Fig. 8b.

We can also observe the average delay increasing with $P_{r k}$ in Fig. 8b. High values of $P_{r k}$ curtails the vehicle from choosing new radio resources for transmission. When $P_{r k}$ is low, a vehicle again receives more opportunities to encounter the waiting interval (average duration of $\Gamma / 2 \mathrm{~ms}$ ), compared to the longer RRI intervals (duration of $\Gamma \mathrm{ms}$ ). Thus, the variable $P_{r k}$, which is a parameter in the SPS algorithm, can be varied to adjust the average delay in a network that utilizes $\mathrm{C}-\mathrm{V} 2 \mathrm{X}$ Mode 4 for communication. The high $P_{r k}$ values lead to higher average delays, but a reduction in jitter as the RRI intervals stay more homogeneous.

Further practical insights can be drawn by referring to the delay requirements stated for ETSI ITS use cases in [32]. For example, a vehicle's emergency electronic brake warning or a stationary vehicle warning requires a minimum of $10 \mathrm{~Hz}$ frequency and a maximum delay of $100 \mathrm{~ms}$. On the other hand, a less safety-critical scenario such as road works requires a minimum frequency of $2 \mathrm{~Hz}$ and a maximum delay of 100 ms. When considering all scenarios, we can conclude that a maximum delay of less than $100 \mathrm{~ms}$ is desirable for safety. Based on our results for C-V2X Mode 4, it can be seen that $\Gamma=20 \mathrm{~ms}$ and $\Gamma=50 \mathrm{~ms}$ can both satisfy this delay constraint on the average, at the highest CAM frequency of $10 \mathrm{~Hz}$. However, as illustrated in Fig. 8a, $\Gamma=100 \mathrm{~ms}$ fails to satisfy this delay requirement on the average, at the highest CAM rate of $10 \mathrm{~Hz}$. On the other hand, as shown in Fig. 7a, we can observe that the delay constraint is satisfied on the average for almost all parameter settings in IEEE 802.11p. Although the average delay increases with $N$, it can be observed that a system employing IEEE 802.11p for communication can support approximately 1000 vehicles without violating the 100 ms delay constraint on the average. The network can be made further dense by utilizing adaptive CAM.

2) Collision probability: As shown in Fig. 9a, it is not surprising that the collision probability increases with $N$ in both C-V2X Mode 4 and IEEE 802.11p. However, a vehicle that utilizes C-V2X Mode 4 has a lower collision probability than a vehicle that utilizes IEEE 802.11p. Thus, the SPS algorithm performs better in terms of collision resolution compared to the contention-based method in IEEE 802.11p. Therefore, C-V2X Mode 4 is a better choice for use cases that insist on ultra-reliable communications. We can observe that the collision probability in C-V2X Mode 4 increases with $\Gamma$. Higher values of $\Gamma$ lead to longer selection windows, which increases the chances of two or more selection windows overlapping, as explained with regards to Fig. 6. It can be

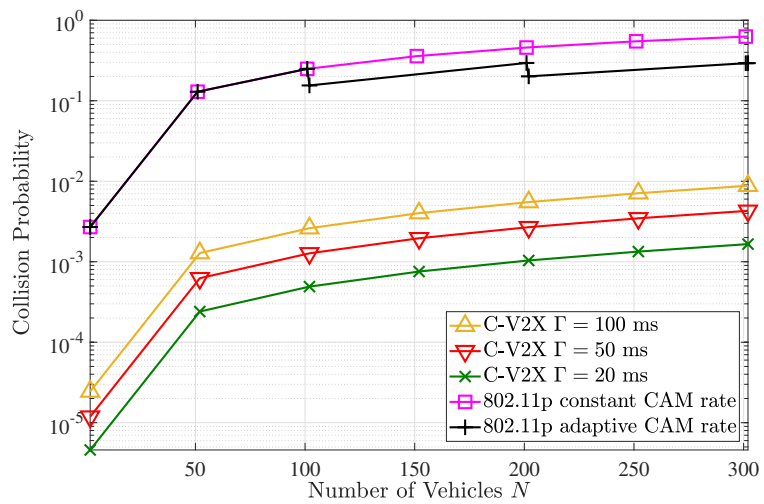

(a) Collision probability vs $N$.

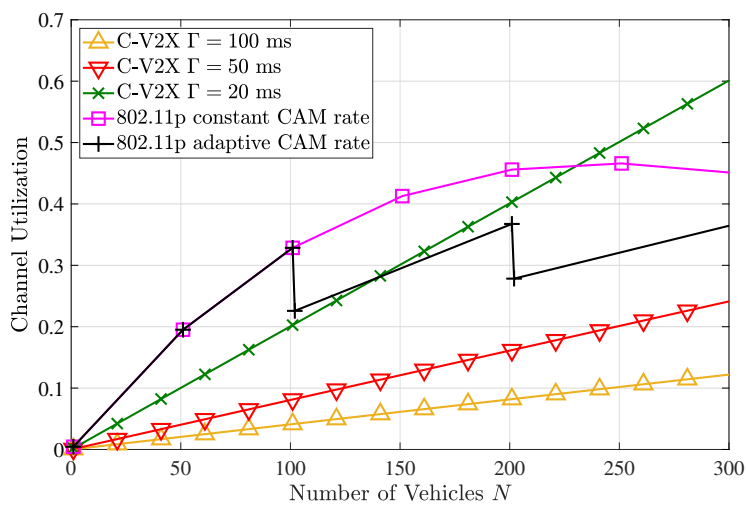

(b) Average channel utilization vs $N$.

Figure 9: The behavior of the collision probability and the average channel utilization with $N$, where $\lambda=1, T_{C}=T_{D}=100 \mathrm{~ms}, P_{r k}=0.4$, and $K=5$.

observed that the adaptive CAM rate alleviates the collision probability of IEEE 802.11 p marginally, but the collision rate is high when $N>50$. The behavior of the collision probability with $T_{C}$ is similar to what was observed for the average delay in Fig. 8a. That is, it decreases monotonically with $T_{C}$ for IEEE 802.11p, and it decreases first and then increases with a local optimum point for C-V2X. However, although the behavior for $\mathrm{C}-\mathrm{V} 2 \mathrm{X}$ is similar, the variation with $T_{C}$ in terms of magnitude is negligibly small.

3) Average channel utilization: As illustrated in Fig. 9b, the average channel utilization of C-V2X Mode 4 increases almost linearly with $N$. The rate at which the average channel utilization increases, decreases with the value of $\Gamma$. The system also exhibits lower average channel utilization for longer selection window sizes. In IEEE 802.11p, the average channel utilization increases with $N$ up to about 200 and then saturates due to reaching the contention access based conditional stability region boundary. The average channel utilization of IEEE $802.11 \mathrm{p}$ can be improved with adaptive packet arrival. However, in general, it is always higher than C-V2X Mode 4. This shows that selecting a proper repetition interval for CAM packets can also be used for better channel utilization in IEEE 802.11p. The average channel utilization of both technologies decreases monotonically with $T_{C}$ as the number of packets transmitted per second reduces when $T_{C}$ increases. Similar behavior can be observed in the channel busy ratio. To this end, for $N=300, \lambda=1$ packet/s, 
$T_{D}=100 \mathrm{~ms}, P_{r k}=0.4$ and $K=5$, the reduction in average channel utilization when $T_{C}$ is increased from $100 \mathrm{~ms}$ to $1 \mathrm{~s}$ is $17.95 \%$ for IEEE $802.11 \mathrm{p}$. For C-V2X, this reduction is significantly small, e.g., for $\Gamma=100 \mathrm{~ms}$, the reduction is $2.80 \%$.

\section{CONCLUSIONS}

This paper has presented multi-dimensional DTMC models to compare the MAC layer performance of the ETSI ITSG5 IEEE 802.11p and C-V2X Mode 4, considering CAM and DENM packets proposed for ITS. DTMC based traffic generators and a device-level queue model have been used to feed the packets to the aforementioned DTMCs for transmission. Closed-form solutions for the steady-state probabilities of the models have been obtained, and they have been then utilized to derive expressions for key MAC layerspecific performance indicators such as the average delay, the collision probability, and the average channel utilization. An application of the models has been used for numerical results. The results have shown how the performance metrics of each communication technology vary for different parameter selections. When comparing the two technologies, the average delay of C-V2X Mode 4 is comparatively higher than IEEE 802.11 p. On the other hand, the collision probability of a vehicle communicating using $\mathrm{C}-\mathrm{V} 2 \mathrm{X}$ Mode 4 is lower than its counterpart. The results have also shown that the average delay of C-V2X has a locally optimal combination of CAM and DENM packet arrival rates, which can be utilized to reduce delays in C-V2X further. Moreover, the DCC algorithm's TRC technique can be used to regulate the collision probability and the channel utilization of a vehicle communicating using IEEE 802.11p.

\section{APPENDIX A}

\section{DERIVATIONS OF COLLISION PROBABILITY}

\section{A. Proof of Lemma 1}

The selection window initiates at reaching state $(1,0)$, and this is the scenario where a collision can occur. The cycle time of state $(1,0)$ is $1 / \pi_{1,0}$. Consider that vehicle $v$ initiated its selection window. The probability of a neighboring vehicle reaching state $(1,0)$ during vehicle $v$ 's selection window is given by $1-\prod_{i=0}^{\Gamma-1}\left(1-\frac{1}{1 / \pi_{1,0}-i}\right)=\tilde{p}$. Similarly, the probability of a neighboring vehicle reaching state $(1,0)$ during vehicle $v$ 's selection window and selecting the same CSR as vehicle $v$ is given by $\tilde{p}\left(1-P_{r k}\right) /\left(C S R_{t o t}-C S R_{e x c}\right)$, where $C S R_{\text {exc }}$ denotes the number of CSRs excluded according to the SPS algorithm such that there are $C S R_{t o t}-C S R_{e x c}$ CSRs in $L_{1}$. Thus, the probability of all $N-1$ neighboring vehicles not selecting the same CSR as vehicle $v$ is given by $\left[1-\tilde{p}\left(1-P_{r k}\right) /\left(C S R_{t o t}-C S R_{e x c}\right)\right]^{N-1}$, and $\left.1-\left[1-\tilde{p}\left(1-P_{r k}\right) / C S R_{t o t}-C S R_{e x c}\right)\right]^{N-1}$ gives us the collision probability.

Let $\xi$ denote the ratio between the size of the sensing window and the selection window, and $\Phi$ denote the number of times we encounter $R C=1$ in a given sensing window. Since $\xi \leq 2 R_{l}$ according to the standard [10], we have
$\Phi \in\{0,1,2\}$. This means, depending on the value of $\Phi$, the vehicle of interest $v$ may either use 1 CSR, 2 CSRs or 3 CSRs. Thus, the average number of CSRs used by vehicle $v$ is given by $P_{1}+2 P_{2}+3 P_{3}$, where $P_{i}$ is the probability of using $i$ CSR values. The number of CSRs used by the neighboring vehicles known through SCI is approximately $N-1$. Hence, $C S R_{\text {exc }} \approx N-1+P_{1}+2 P_{2}+3 P_{3}$. It is not hard to see that $P_{1}+2 P_{2}+3 P_{3} \leq 3<<N-1$. Thus, we consider $C S R_{\text {exc }} \approx N-1$, which completes the proof.

\section{B. Proof of Lemma 2}

Let $P_{\text {suc }}=\operatorname{Pr}\{$ exactly one vehicle transmits $\mid$ at least one vehicle transmits\}, which can be simplified as the ratio between $P_{\text {suc }}=\operatorname{Pr}\{$ exactly one vehicle transmits $\}$ and $\operatorname{Pr}\{$ at least one vehicle transmits $\}$. Successful transmission of a packet by vehicle $v$ can be obtained from the steady-state probabilities of the DTMC model for IEEE $802.11 \mathrm{p}$ as $(1-\theta)\left(\pi_{I, 0}+\pi_{A_{\Omega}}\right)+\sum_{j=1}^{\vartheta} \pi_{T x, j}$. Similarly, the probability of the $N-1$ neighbors not transmitting is given by $\left[1-\left(\pi_{I, 0}+\pi_{A_{\Omega}}+\sum_{j=1}^{\vartheta} \pi_{T x, j}\right)\right]^{(N-1)}$. Thus, the probability of exactly one vehicle transmitting is given by $N\left[(1-\theta)\left(\pi_{I, 0}+\pi_{A_{\Omega}}\right)+\sum_{j=1}^{\vartheta} \pi_{T x, j}\right][1-$ $\left.\left(\pi_{I, 0}+\pi_{A_{\Omega}}+\sum_{j=1}^{\vartheta} \pi_{T x, j}\right)\right]^{(N-1)}$, and the probability of at least one vehicle transmitting is given by 1 $\left[1-\left(\pi_{I, 0}+\pi_{A_{\Omega}}+\sum_{j=1}^{\vartheta} \pi_{T x, j}\right)\right]^{N}$. The ratio of these probabilities gives us $P_{\text {suc }}$, and $P_{\text {col }}^{11 p}=1-P_{\text {suc }}$ completes the proof.

\section{APPENDIX B}

\section{DERIVATIONS OF AVERAGE DELAY}

\section{A. Proof of Lemma 3}

From the steady-state probabilities of the queue model, $1 / P_{t x o}$ is the duration in milliseconds (cycle time) to serve one packet. For the first packet, we may not spend the total cycle time to serve the packet, as it depends on the state vehicle $v$ is in. Thus, we consider the service time to be $\frac{1}{2 P_{t x o}}$ (half the cycle time) for the first packet. From the second packet onwards, we add $\frac{1}{P_{t x o}}$ to the service time of the previous packet to obtain the delay. For example, the service times of the second and the third packets are calculated as $\frac{3}{2 P_{t x o}}$ and $\frac{5}{2 P_{t x o}}$, respectively. We consider a queue of length $M$, and the averaging is done by utilizing the steadystate probability of each state, conditioned on the fact that the queue is not empty. Thus, the average delay is given by $d_{\text {ave }}^{v 2 x}=\sum_{i=1}^{M} \frac{2 i-1}{2 P_{\text {txo }}} \pi_{i} /\left(1-P_{q e}\right)$, which completes the proof.

\section{B. Proof of Lemma 4}

Since unit time is considered to be aSlotTime, the delay associated with the transmit states is $\vartheta$. The delay associated with states $A_{i}$ where $i \in\{1, \ldots, \Omega\}$ is $1+\sum_{i=1}^{\Omega-1}(1-\theta)^{i}$. The delay associated with the remaining states, i.e., state $i \in S^{11 p}-\left\{I d l e, \bigcup_{j=1}^{\vartheta} T x, j, \bigcup_{i=1}^{\Omega} A_{i}\right\}$ can be calculated by utilizing the product of the corresponding delay of each state $\left(D_{i}\right)$ with the steady-state probability of each state conditioned 
on the fact that $i \in S^{11 p}-\left\{I d l e, \bigcup_{j=1}^{\vartheta} T x, j, \bigcup_{i=1}^{\Omega} A_{i}\right\}$. Sum of the three delay values completes the proof.

\section{REFERENCES}

[1] IEEE, "IEEE standard for information technology- local and metropolitan area networks- specific requirements- part 11: Wireless LAN medium access control (MAC) and physical layer (PHY) specifications amendment 6: Wireless access in vehicular environments," IEEE Std 802.11p-2010, pp. 1-51, Jul. 2010.

[2] IEEE, "IEEE standard for information technology-telecommunications and information exchange between systems local and metropolitan area networks-specific requirements part 11: Wireless LAN medium access control (MAC) and physical layer (PHY) specifications," IEEE Std 802.11-2012, pp. 1-2793, Mar. 2012.

[3] IEEE, "IEEE standard for information technology-telecommunications and information exchange between systems local and metropolitan area networks - specific requirements - part 11: Wireless LAN medium access control (MAC) and physical layer (PHY) specifications," IEEE Std 802.11-2016, pp. 1-3534, Dec. 2016.

[4] ETSI, "Intelligent transport systems (ITS); vehicular communications; basic set of applications; part 2: Specification of cooperative awareness basic service," Technical Specification (TS) 102 637-2, European Telecommunications Standards Institute (ETSI), Mar. 2011, version 1.2.1.

[5] ETSI, "Intelligent transport systems (ITS); vehicular communications: basic set of applications; part 3: Specification of decentralized environmental notification basic service," Technical Specification (TS) 102 637-3, European Telecommunications Standards Institute (ETSI), Sep. 2010, version 1.1.1.

[6] ETSI, "Intelligent transport systems (ITS); Access layer specification for intelligent transport system operating in the $5 \mathrm{GHz}$ frequency band," European Standard telecommunications series (EN) 302 663, European Telecommunications Standards Institute (ETSI), May 2013, version 1.2.1.

[7] ETSI, "Intelligent transport systems (ITS); Vehicular communications; geonetworking; part 4: Geographical addressing and forwarding for point-to-point and point-to-multipoint communications; sub-part 2: Media-dependent functionalities for ITS-G5," Technical Specification (TS) 102 636-4-2, European Telecommunications Standards Institute (ETSI), Oct. 2013, version 1.1.1.

[8] 3GPP, "Evolved universal terrestrial radio access (E-UTRA) and evolved universal terrestrial radio access network (E-UTRAN); Overall description; stage 2," Technical Specification (TS) 36.300, 3rd Generation Partnership Project (3GPP), Dec. 2016, version 14.1.0.

[9] 3GPP, "Evolved universal terrestrial radio access (E-UTRA); Physical layer procedures," Technical Specification (TS) 36.213, 3rd Generation Partnership Project (3GPP), Apr. 2017, version 14.2.0.

[10] 3GPP, "Evolved universal terrestrial radio access (E-UTRA); Medium access control (MAC) protocol specification," Technical Specification (TS) 36.321, 3rd Generation Partnership Project (3GPP), Jul. 2017, version 14.3.0.

[11] R. Molina-Masegosa and J. Gozalvez, "LTE-V for sidelink 5G V2X vehicular communications: A new $5 \mathrm{G}$ technology for short-range vehicleto-everything communications," IEEE Veh. Technol. Mag., vol. 12, pp. 30-39, Dec. 2017

[12] M. Wang et al., "Comparison of LTE and DSRC-based connectivity for intelligent transportation systems," in Proc. IEEE Vehicular Technology Conference, Sydney, Australia, pp. 1-5, Jun. 2017.

[13] T. V. Nguyen et al., "A comparison of cellular vehicle-to-everything and dedicated short range communication," in Proc. IEEE Vehicular Networking Conference, Torino, Italy, pp. 101-108, Nov. 2017.

[14] A. Bazzi, B. M. Masini, A. Zanella, and I. Thibault, "On the performance of IEEE $802.11 \mathrm{p}$ and LTE-V2V for the cooperative awareness of connected vehicles," IEEE Trans. Veh. Technol., vol. 66, pp. 1041910432, Nov. 2017.

[15] V. Vukadinovic et al., "3GPP C-V2X and IEEE 802.11p for vehicleto-vehicle communications in highway platooning scenarios," Ad Hoc Netw., vol. 74, pp. 17-29, 2018.

[16] J. Thota, N. F. Abdullah, A. Doufexi, and S. Armour, "Performance of car to car safety broadcast using cellular V2V and IEEE 802.11p," in Proc. IEEE Vehicular Technology Conference, Porto, Potugal, pp. 1-5, Jun. 2018
[17] W. Anwar, K. Kulkarni, T. R. Augustin, N. Franchi, and G. Fettweis, "PHY abstraction techniques for IEEE 802.11p and LTE-V2V: Applications and analysis," in Proc. IEEE Globecom Workshops, Abu Dhabi, UAE, pp. 1-7, Dec. 2018.

[18] G. Cecchini, A. Bazzi, B. M. Masini, and A. Zanella, "Performance comparison between IEEE $802.11 \mathrm{p}$ and LTE-V2V in-coverage and out-of-coverage for cooperative awareness," in Proc. IEEE Vehicular Networking Conference, Torino, Italy, pp. 109-114, Nov. 2017.

[19] S. Kuehlmorgen, P. Schmager, A. Festag, and G. Fettweis, "Simulationbased evaluation of ETSI ITS-G5 and Cellular-VCS in a real-world road traffic scenario," in Proc. IEEE Vehicular Technology Conference, Chicago, USA, pp. 1-6, Aug. 2018.

[20] Y. Jeon, S. Kuk, and H. Kim, "Reducing message collisions in sensingbased semi-persistent scheduling (SPS) by using reselection lookaheads in cellular V2X," Sensors, vol. 18, p. 4388, Dec. 2018.

[21] M. Gonzalez-Martín, M. Sepulcre, R. Molina-Masegosa, and J. Gozalvez, "Analytical models of the performance of C-V2X Mode 4 vehicular communications," IEEE Trans. Veh. Technol., vol. 68, pp. 1155-1166, Feb. 2019.

[22] G. P. Wijesiri N.B.A., J. Haapola, and T. Samarasinghe, "A Markov perspective on C-V2X Mode 4," in Proc. IEEE Vehicular Technology Conference, Hawaii, USA, pp. 1-6, Sep. 2019.

[23] Y. Y. Nasrallah, I. Al-Anbagi, and H. T. Mouftah, "A realistic analytical model of IEEE $802.11 \mathrm{p}$ for wireless access in vehicular networks," in Proc. International Conference on Connected Vehicles and Expo, Vienna, Austria, pp. 1029-1034, Nov. 2014.

[24] F. Romeo, C. Campolo, A. Molinaro, and A. O. Berthet, "DENM repetitions to enhance reliability of the autonomous mode in NR V2X sidelink," in Proc. IEEE Vehicular Technology Conference, Antwerp, Belgium, pp. 1-5, May 2020.

[25] G. Naik, B. Choudhury, and J. Park, "IEEE 802.11bd 5G NR V2X: Evolution of radio access technologies for $\mathrm{V} 2 \mathrm{X}$ communications," IEEE Access, vol. 7, pp. 70169-70184, May 2019.

[26] A. Bazzi, G. Cecchini, M. Menarini, B. M. Masini, and A. Zanella, "Survey and perspectives of vehicular Wi-Fi versus sidelink cellularV2X in the 5G era," Future Internet, vol. 11, p. 122, May 2019.

[27] C. D. Manning, P. Raghavan, and H. Schutze, Introduction to Information Retrieval. Cambridge University Press, 2008.

[28] N. Bouchemal, R. Naja, and S. Tohme, "Traffic modeling and performance evaluation in vehicle to infrastructure $802.11 \mathrm{p}$ network," in $\mathrm{Ad}$ Hoc Networks, Springer International Publishing, pp. 82-99, 2014.

[29] G. Bianchi, "Performance analysis of the IEEE 802.11 distributed coordination function," IEEE J. Sel. Areas Commun., vol. 18, pp. 535547, Mar. 2000.

[30] J. Santa, F. Pereñíguez, A. Moragón, and A. F. Skarmeta, "Vehicleto-infrastructure messaging proposal based on CAM/DENM specifications," in Proc. IFIP Wireless Days, Valencia, Spain, pp. 1-7, Nov. 2013.

[31] D. Martín-Sacristán et al., "Evaluation of LTE-Advanced connectivity options for the provisioning of V2X services," in Proc. IEEE Wireless Communications and Networking Conference, Barcelona, Spain, pp. 16, Apr. 2018.

[32] ETSI, "Intelligent transport systems (ITS); vehicular communications; Basic Set of Applications; Definitions," Technical Report (TR) 102638 , European Telecommunications Standards Institute (ETSI), Jun. 2009, version 1.1.1.

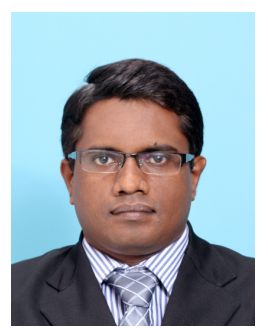

Geeth P. Wijesiri N. B. A. (M'18) was born in Galle, Sri Lanka, in 1982. He received the B.Sc. Engineering (First Class Honors) degree from the Department of Electrical and Information Engineering, University of Ruhuna, Sri Lanka, in 2008 and worked as a Senior Engineer at Lanka Bell Ltd, Sri Lanka for five years $(2008-2013)$. Then he received his M.Sc. Degree in Information and Communication Technology from the University of Agder, Norway, in 2016. Then, he joined the Department of Electrical and Information Engineering, University of Ruhuna, Sri Lanka, as a lecture in 2016 (currently on study leave). Since May 2018, he has been with the Department of Electronic and Telecommunication Engineering, University of Moratuwa, Sri Lanka, where he is a Ph.D. student. His research interests are in computer networks, vehicular communications, and wireless networks. 


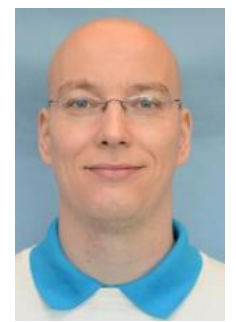

Jussi Haapola, adjunct professor, received his M.Sc. and $\mathrm{Ph} . \mathrm{D}$. degrees from the University of Oulu, Finland, in 2002 from Physical Sciences and in 2010 from Telecommunications Engineering, respectively. He joined the Centre for Wireless Communications at the University of Oulu in 2001, where he currently works as a Senior Research Fellow on heterogeneous IoT and cellular systems for vertical applications. In 2011 he made a Post-doctoral Fellow visit in Tohoku University, Sendai, Japan and between 2012 and 2015 he also worked for University of Oulu Research Institute Japan - CWC-Nippon Ltd as Deputy manager. He is currently the coordinator of EC H2020 project 5G!Drones (No. 857031).

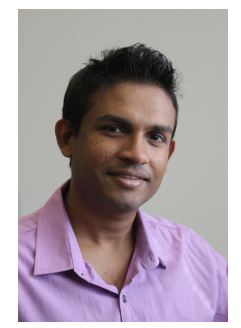

Tharaka Samarasinghe (S'11-M'13) was born in Colombo, Sri Lanka, in 1984. He received the B.Sc. degree in engineering from the Department of Electronic and Telecommunication Engineering, University of Moratuwa, Sri Lanka, in 2008, where he received the award for the most outstanding undergraduate upon graduation. He received the Ph.D. degree from the Department of Electrical and Electronic Engineering, University of Melbourne, Australia, in 2012. He was a Research Fellow at the Department of Electrical and Computer Systems Engineering, Monash University, Australia, from 2012 to 2014. He has been with the Department of Electronic and Telecommunication Engineering, University of Moratuwa, Sri Lanka, since January 2015, where he is a Senior Lecturer. He is also an honorary fellow at the Department of Electrical and Electronic Engineering, University of Melbourne, Australia. His research interests are in communications theory, information theory, and wireless networks. 